\title{
Some Notions on Interval-Valued Neutrosophic Sets
}

\author{
Y1ldıray Çelik ${ }^{1 *}$ \\ $\mathbf{1}^{*}$ Ordu University, Faculty of Science and Arts, Departmant of Mathematics, Ordu, Turkey, (ORCID: 0000-0003-3373-3916) \\ ycelik61@gmail.com
}

(1st International Conference on Applied Engineering and Natural Sciences ICAENS 2021, November 1-3, 2021)

(DOI: 10.31590/ejosat.1011428)

ATIF/REFERENCE: Çelik, Y. (2021). Some Notions on Interval-Valued Neutrosophic Sets. European Journal of Science and Technology, (28), 802-805.

\begin{abstract}
In this study, we give some new notions such as intersection, union and complement on interval-valued neutrosophic sets and introduce basic properties related these notions.
\end{abstract}

Keywords: Fuzzy set, Intuitionistic fuzzy set, Interval-valued fuzzy set, Neutrosophic set, Interval-valued neutrosophic set.

\section{Aralık-Değerli Neutrosophic Kümeler Üzerinde Bazı Kavramlar}

Öz

Biz bu çalışmada aralık-değerli neutrosophic kümeler üzerinde arakesit, birleşim ve tümleyen gibi bazı yeni kavramlar verdik ve bu kavramlar ile ilişkili temel özellikleri inceledik.

Anahtar Kelimeler: Bulanık küme, Sezgisel bulanık küme, Aralık-değerli bulanık küme, Neutrosophic küme, Aralık-değerli neutrosophic küme. 


\section{Introduction}

Fuzzy set theory [1] was put forward to develop solutions to problems involving uncertainty. A fuzzy set is characterized with the help of a membership function. In the set $A$, the degree of belonging of an element to the set is $\mu_{A}(x)$, while the degree of non-belonging is naturally $1-\mu_{A}(x)$. Thus, the sum of the degrees of belonging and non-belonging is equal to 1 . However, this approach is not an effecctive method in dealing with uncertainty in real life applications. Because the sum of the degrees of belonging and non-belonging can be less than one. For this reason, Atanassov [2], by adding the nonmembership function to the membership function in fuzzy sets, introduced the intuitionistic fuzzy set theory which is a generalized version of the fuzzy set theory for more precise measurements of belonging. By adding the uncertainty function as a third component to the membership function and the non membership function, neutrosophic set theory which is a special case of fuzzy logic introduced by Smarandache [3]. In neutrosophic sets, the correct membership function and the non-membership function are independent from each other, and this situation making it more flexible and more realistic than modeling using intuitionistic fuzzy sets. Wang et al. [4] defined the concept of a single valued neutrosophic set for convenience in applications. For precise membership measurements, Zhang et al. [5] extended singlevalued neutrosophic sets to interval-valued neutrosophic sets and described some operations related to these sets. Lupianez [6] examined the relations between interval neutrosophic sets and topology. In our study, some notions are defined on intervalvalued neutrosophic sets (ivns). Then, considering these defined notions, some fundamental theorems for interval valued neutrosophic sets (ivns) are proved.

\section{Preliminaries}

Definition 2.1 [1] Let $X \neq \emptyset$ be a set. The function $\mu: X \rightarrow[0,1]$ is called fuzzy subset and is defined by $\mu=\{(\alpha, \mu(\alpha)): \alpha \in$ $X, \mu(\alpha) \in[0,1]\}$.

Let $\mu$ and $v$ be two fuzzy subset of $X$. Then,

$\mu \leq v$ if and only if $\mu(\alpha) \leq v(\alpha)$ for all $\alpha \in X$.

Definition 2.2 [7] An interval-valued fuzzy set $\mu$ on the universe $X \neq \emptyset$ is a mapping $\mu: X \rightarrow[0,1]$ such that the membership degree of $\alpha \in X$ is given by $\mu(\alpha)=\left[\mu(\alpha)^{l}, \mu(\alpha)^{u}\right] \subseteq[0,1]$, where $\mu(\alpha)^{l}$ and $\mu(\alpha)^{u}$ denotes the lower and the upper bound of $\mu(\alpha)$, respectively.

Definition 2.3 [8] A neutrosophic set $A$ on the universe $X$ is defined as

$A=\left\{\left\langle\alpha, T_{A}(\alpha), I_{A}(\alpha), F_{A}(\alpha)\right\rangle, \alpha \in X\right\}$, where the functions $T, I, F: X \rightarrow[0,1]$ define a truth-membership, an indeterminacymembership and a falsity-membership functions of an element $\alpha \in X$ for a set $A$, respectively, with the condition $-0 \leq T_{A}(\alpha)+$ $I_{A}(\alpha)+F_{A}(\alpha) \leq 3^{+}$.

\section{Some Notions on Interval-Valued Neutrosophic Sets}

Definition 3.1 [9] An ivns $\dot{A}$ over $X$ is characterized by truth membership function $T_{\dot{A}}(\alpha)$, indeteminacy membership function $I_{\dot{A}}(\alpha)$ and falsity membership function $F_{\dot{A}}(\alpha)$ of an element $\alpha \in$ $X$ for a set $\dot{A}$, respectively, and defined by $\dot{A}=$ $\left\{\left\langle\alpha, T_{\dot{A}}(\alpha), I_{\dot{A}}(\alpha), F_{\dot{A}}(\alpha)\right\rangle: \alpha \in X\right\}$. For all $\alpha \in X$, one has that

$T_{\dot{A}}(\alpha)=\left[T_{\dot{A}}^{l}(\alpha), T_{\dot{A}}^{u}(\alpha)\right]$

$I_{\dot{A}}(\alpha)=\left[I_{\dot{A}}^{l}(\alpha), I_{\dot{A}}^{u}(\alpha)\right]$

$F_{\dot{A}}(\alpha)=\left[F_{\dot{A}}^{l}(\alpha), F_{\dot{A}}^{u}(\alpha)\right]$

The family of all ivns over $X$ is showed by $\operatorname{IVN}(X)$.

Definition 3.2 Let $\dot{A} \in \operatorname{IVN}(X)$. Then,

i. If $\dot{A}=\{\langle\alpha,[0,0],[1,1],[1,1]\rangle \mid \alpha \in X\}$, then $\dot{A}$ is called empty ivns and denoted by $\dot{\varnothing}$.

ii. If $\dot{A}=\{\langle\alpha,[1,1],[0,0],[0,0]\rangle \mid \alpha \in X\}$, then $\dot{A}$ is called totally ivns and denoted by $\dot{\Omega}$.

iii. The complement of $\dot{A}$ is defined by

$\overline{\dot{A}}=\left\{\left\langle\alpha,\left[F_{\dot{A}}^{l}(\alpha), F_{\dot{A}}^{u}(\alpha)\right],\left[1-I_{\dot{A}}^{u}(\alpha), 1-I_{\dot{A}}^{l}(\alpha)\right]\right.\right.$,

$\left.\left.\left[T_{\dot{A}}^{l}(\alpha), T_{\dot{A}}^{u}(\alpha)\right]\right\rangle \mid \alpha \in X\right\}$

for all $\alpha \in X$.

Definition 3.3 Let $\dot{A}, \dot{B} \in \operatorname{IVN}(X)$. Then $\dot{A}$ is said to be ivn subset of $\dot{B}$ iff

$T_{\dot{A}}^{l}(\alpha) \leq T_{\dot{B}}^{l}(\alpha) \quad T_{\dot{A}}^{u}(\alpha) \leq T_{\dot{B}}^{u}(\alpha)$

$I_{\dot{A}}^{l}(\alpha) \geq I_{\dot{B}}^{l}(\alpha) \quad I_{\dot{A}}^{u}(\alpha) \geq I_{\dot{B}}^{u}(\alpha)$

$F_{\dot{A}}^{l}(\alpha) \geq F_{\dot{B}}^{l}(\alpha) \quad F_{\dot{A}}^{u}(\alpha) \geq F_{\dot{B}}^{u}(\alpha)$

for all $\alpha \in X$. This situation is denoted by $\dot{A} \widehat{\subseteq} \dot{B}$.

Definition 3.4 Let $\dot{A}, \dot{B} \in \operatorname{IVN}(X)$. Then,

i. The intersection of $\dot{A}$ and $\dot{B}$ is defined by

$\dot{A} \widehat{\cap} \dot{B}=\left\{\left\langle\alpha,\left[\min \left\{T_{\dot{A}}^{l}(\alpha), T_{\dot{B}}^{l}(\alpha)\right\}, \min \left\{T_{\dot{A}}^{+}(\alpha), T_{\dot{B}}^{+}(\alpha)\right\}\right]\right.\right.$,

$$
\begin{aligned}
& {\left[\max \left\{I_{\dot{A}}^{l}(\alpha), I_{\dot{B}}^{l}(\alpha)\right\}, \max \left\{I_{\dot{A}}^{+}(\alpha), I_{\dot{B}}^{+}(\alpha)\right\}\right],} \\
& \left.\left[\max \left\{F_{\dot{A}}^{l}(\alpha), F_{\dot{B}}^{l}(\alpha)\right\}, \max \left\{F_{\dot{A}}^{u}(\alpha), F_{\dot{B}}^{u}(\alpha)\right\}\right]\right\rangle
\end{aligned}
$$

$\mid \alpha \in X\}$

for all $\alpha \in X$.

ii. The union of $\dot{A}$ and $\dot{B}$ is defined by

$\dot{A} \widehat{\cup} \dot{B}=\left\{\left\langle\alpha,\left[\max \left\{T_{\dot{A}}^{l}(\alpha), T_{\dot{B}}^{l}(\alpha)\right\}, \max \left\{T_{\dot{A}}^{+}(\alpha), T_{\dot{B}}^{+}(\alpha)\right\}\right]\right.\right.$,

$$
\begin{aligned}
& {\left[\min \left\{I_{\dot{A}}^{l}(\alpha), I_{\dot{B}}^{l}(\alpha)\right\}, \min \left\{I_{\dot{A}}^{+}(\alpha), I_{\dot{B}}^{+}(\alpha)\right\}\right],} \\
& \left.\left[\min \left\{F_{\dot{A}}^{l}(\alpha), F_{\dot{B}}^{l}(\alpha)\right\}, \min \left\{F_{\dot{A}}^{u}(\alpha), F_{\dot{B}}^{u}(\alpha)\right\}\right]\right\rangle
\end{aligned}
$$

$\mid \alpha \in X\}$

for all $\alpha \in X$.

It is clearly seen that intersection and union of two ivns are ivns.

Theorem 3.5 Let $\dot{A} \in I V N(X)$. Then, 
i. $\dot{A} \hat{\cap} \dot{\emptyset}=\dot{\emptyset}, \quad \dot{A} \hat{\cap} \dot{\Omega}=\dot{A}$

ii. $\dot{A} \widehat{\cup} \dot{\emptyset}=\dot{A}, \dot{A} \widehat{0} \dot{\Omega}=\dot{\Omega}$

Proof. Let's consider ivns $\dot{A}$ on $X$ as follows.

$\dot{A}=\left\{\left\langle\alpha,\left[T_{\dot{A}}^{l}(\alpha), T_{\dot{A}}^{u}(\alpha)\right)\right],\left[I_{\dot{A}}^{l}(\alpha), I_{\dot{A}}^{u}(\alpha)\right]\right.$,

$$
\left.\left.\left[F_{\dot{A}}^{l}(\alpha), F_{\dot{A}}^{u}(\alpha)\right]\right\rangle: \alpha \in X\right\}
$$

i. For all $\alpha \in X$,

$\dot{A} \hat{\cap} \dot{\varnothing}=\left\{\left\langle\alpha,\left[\min \left\{T_{\dot{A}}^{l}(\alpha), T_{\dot{\emptyset}}^{l}(\alpha)\right\}, \min \left\{T_{\dot{A}}^{u}(\alpha), T_{\dot{\phi}}^{u}(\alpha)\right]\right\}\right.\right.$,

$$
\begin{aligned}
& {\left[\max \left\{I_{\dot{A}}^{l}(\alpha), I_{\dot{\phi}}^{l}(\alpha)\right\}, \max \left\{I_{\dot{A}}^{u}(\alpha), I_{\dot{\phi}}^{u}(\alpha)\right\}\right],} \\
& \left.\left[\max \left\{F_{\dot{A}}^{l}(\alpha), F_{\dot{\phi}}^{l}(\alpha)\right\}, \max \left\{F_{\dot{A}}^{u}(\alpha), F_{\dot{\phi}}^{u}(\alpha)\right\}\right]\right\rangle
\end{aligned}
$$

$\mid \alpha \in X\}$

$=\left\{\left\langle\alpha,\left[\min \left\{T_{\dot{A}}^{l}(\alpha), 0\right\}, \min \left\{T_{\dot{A}}^{u}(\alpha), 0\right]\right\}\right.\right.$,

$$
\begin{aligned}
& {\left[\max \left\{I_{\dot{A}}^{l}(\alpha), 1\right\}, \max \left\{I_{\dot{A}}^{u}(\alpha), 1\right\}\right],} \\
& \left.\left[\max \left\{F_{\dot{A}}^{l}(\alpha), 1\right\}, \max \left\{F_{\dot{A}}^{u}(\alpha), 1\right\}\right]\right\rangle
\end{aligned}
$$

$\mid \alpha \in X\}$

$=\{\langle\alpha,[0,0],[1,1],[1,1]\rangle \mid \alpha \in X\}=\dot{\emptyset}$

The case $\dot{A} \widehat{\cap} \dot{\Omega}=\dot{A}$ can be shown similarly.

ii. For all $\alpha \in X$,

$\dot{A} \widehat{U} \dot{\Omega}=\left\{\left\langle\alpha,\left[\max \left\{T_{\dot{A}}^{l}(\alpha), T_{\dot{\Omega}}^{l}(\alpha)\right\}, \max \left\{T_{\dot{A}}^{+}(\alpha), T_{\dot{\Omega}}^{+}(\alpha)\right]\right\}\right.\right.$,

$$
\begin{aligned}
& {\left[\min \left\{I_{\dot{A}}^{l}(\alpha), I_{\dot{\Omega}}^{l}(\alpha)\right\}, \min \left\{I_{\dot{A}}^{u}(\alpha), I_{\dot{\Omega}}^{u}(\alpha)\right\}\right],} \\
& \left.\left[\min \left\{F_{\dot{A}}^{l}(\alpha), F_{\dot{\Omega}}^{l}(\alpha)\right\}, \min \left\{F_{\dot{A}}^{u}(\alpha), F_{\dot{\Omega}}^{u}(\alpha)\right\}\right]\right\rangle
\end{aligned}
$$

$\mid \alpha \in X\}$

$=\left\{\left\langle\alpha,\left[\max \left\{T_{\dot{A}}^{l}(\alpha), 1\right\}, \max \left\{T_{\dot{A}}^{u}(\alpha), 1\right]\right\}\right.\right.$,

$$
\begin{aligned}
& {\left[\min \left\{I_{\dot{A}}^{l}(\alpha), 0\right\}, \min \left\{I_{\dot{A}}^{u}(\alpha), 0\right\}\right],} \\
& \left.\left[\min \left\{F_{\dot{A}}^{l}(\alpha), 0\right\}, \min \left\{F_{\dot{A}}^{u}(\alpha), 0\right\}\right]\right\rangle
\end{aligned}
$$

$\mid \alpha \in X\}$

$=\{\langle\alpha,[1,1],[0,0],[0,0]\rangle \mid \alpha \in X\}=\dot{\Omega}$

The case $\dot{A} \widehat{0} \dot{\emptyset}=\dot{A}$ can be shown in a similar way.

Theorem 3.6 Let $\dot{A}, \dot{B}, \dot{C} \in \operatorname{IVN}(X)$. Then,

$$
\begin{aligned}
& \text { i. }(\dot{A} \widehat{\cap} \dot{B}) \widehat{\cap} \dot{C}=\dot{A} \widehat{\cap}(\dot{B} \widehat{\cap} \dot{C}) \\
& \text { ii. }(\dot{A} \widehat{\cup} \dot{B}) \widehat{\cup} \dot{C}=\dot{A} \widehat{\cup}(\dot{B} \widehat{\cup} \dot{C}) \\
& \text { iii. } \dot{A} \widehat{\cap}(\dot{B} \widehat{\cup} \hat{C})=(\dot{A} \widehat{\cap} \dot{B}) \widehat{\cup}(\dot{A} \widehat{\cap} \dot{C}) \\
& \text { iv. } \dot{A} \widehat{\cup}(\dot{B} \widehat{\cap} \dot{C})=(\dot{A} \widehat{\cup} \dot{B}) \widehat{\cap}(\dot{A} \widehat{\cup} \dot{C})
\end{aligned}
$$

Proof. Let's consider ivns $\dot{A}, \dot{B}$ and $\dot{C}$ on $X$ as follows.

$\dot{A}=\left\{\left\langle\alpha,\left[T_{\dot{A}}^{l}(\alpha), T_{\dot{A}}^{u}(\alpha)\right)\right],\left[I_{\dot{A}}^{l}(\alpha), I_{\dot{A}}^{u}(\alpha)\right]\right.$,

$$
\left.\left.\left[F_{\dot{A}}^{l}(\alpha), F_{\dot{A}}^{u}(\alpha)\right]\right\rangle: \alpha \in X\right\}
$$

$\dot{B}=\left\{\left\langle\alpha,\left[T_{\dot{B}}^{l}(\alpha), T_{\dot{B}}^{u}(\alpha)\right],\left[I_{\dot{B}}^{l}(\alpha), I_{\dot{B}}^{u}(\alpha)\right]\right.\right.$,

$\left.\left.\left[F_{\dot{B}}^{l}(\alpha), F_{\dot{B}}^{u}(\alpha)\right]\right\rangle: \alpha \in X\right\}$

$\dot{C}=\left\{\left\langle\alpha,\left[T_{\dot{C}}^{l}(\alpha), T_{\dot{C}}^{u}(\alpha)\right],\left[I_{\dot{C}}^{l}(\alpha), I_{\dot{C}}^{u}(\alpha)\right]\right.\right.$,
$\left.\left.\left[F_{\dot{C}}^{l}(\alpha), F_{\dot{C}}^{u}(\alpha)\right]\right\rangle: \alpha \in X\right\}$

i. For all $\alpha \in X$,

$$
\begin{gathered}
(\dot{A} \hat{\cap} \dot{B}) \hat{\cap} \dot{C}=\left\{\left\langle\alpha,\left[\min \left\{\min \left\{T_{\dot{A}}^{l}(\alpha), T_{\dot{B}}^{l}(\alpha)\right\}, T_{\dot{C}}^{l}(\alpha)\right\},\right.\right.\right. \\
\left.\min \left\{\min \left\{T_{\dot{A}}^{u}(\alpha), T_{\dot{B}}^{u}(\alpha)\right\}, T_{\dot{C}}^{u}(\alpha)\right\}\right], \\
{\left[\max \left\{\max \left\{I_{\dot{A}}^{l}(\alpha), I_{\dot{B}}^{l}(\alpha)\right\}, I_{\dot{C}}^{l}(\alpha)\right\},\right.} \\
\left.\max \left\{\max \left\{I_{\dot{A}}^{u}(\alpha), I_{\dot{B}}^{u}(\alpha)\right\}, I_{\dot{C}}^{u}(\alpha)\right\}\right], \\
{\left[\max \left\{\max \left\{F_{\dot{A}}^{l}(\alpha), F_{\dot{B}}^{l}(\alpha)\right\}, F_{\dot{C}}^{l}(\alpha)\right\},\right.} \\
\left.\left.\left.\max \left\{\max \left\{F_{\dot{A}}^{u}(\alpha), F_{\dot{B}}^{u}(\alpha)\right\}, F_{\dot{C}}^{u}(\alpha)\right\}\right]\right\rangle\right\} \\
=\left\{\left\langle\alpha,\left[\min \left\{T_{\dot{A}}^{l}(\alpha), \min \left\{T_{\dot{B}}^{l}(\alpha), T_{\dot{C}}^{l}(\alpha)\right\}\right\},\right.\right.\right. \\
\left.\min \left\{T_{\dot{A}}^{u}(\alpha), \min \left\{T_{\dot{B}}^{u}(\alpha), T_{\dot{C}}^{u}(\alpha)\right\}\right\}\right], \\
\quad\left[\max \left\{I_{\dot{A}}^{l}(\alpha), \max \left\{I_{\dot{B}}^{l}(\alpha), I_{\dot{C}}^{l}(\alpha)\right\}\right\},\right. \\
\left.\max \left\{I_{\dot{A}}^{u}(\alpha), \max \left\{I_{\dot{B}}^{u}(\alpha), I_{\dot{C}}^{u}(\alpha)\right\}\right\}\right], \\
{\left[\max \left\{F_{\dot{A}}^{l}(\alpha), \max \left\{F_{\dot{B}}^{l}(\alpha), F_{\dot{C}}^{l}(\alpha)\right\}\right\},\right.} \\
\left.\left.\max \left\{F_{\dot{A}}^{u}(\alpha), \max \left\{F_{\dot{B}}^{u}(\alpha), F_{\dot{C}}^{u}(\alpha)\right\}\right\}\right]\right\rangle \\
=\dot{A} \hat{\cap}(\dot{B} \hat{\cap} \dot{C}) \\
\text { ii. It is straightforward. } \\
\text { iii. For all } x \in X,
\end{gathered}
$$

$$
\begin{aligned}
\dot{A} \hat{\cap}(\dot{B} \widehat{\cup} \dot{C})=\{\langle\alpha,[ & \min \left\{T_{\dot{\dot{C}}}^{l}(\alpha), \max \left\{T_{\dot{B}}^{l}(\alpha), T_{\dot{C}}^{l}(\alpha)\right\}\right\}, \\
& \left.\min \left\{T_{\dot{A}}^{u}(\alpha), \max \left\{T_{\dot{B}}^{u}(\alpha), T_{\dot{C}}^{u}(\alpha)\right\}\right\}\right], \\
{[} & \max \left\{I_{\dot{A}}^{l}(\alpha), \min \left\{I_{\dot{B}}^{l}(\alpha), I_{\dot{C}}^{l}(\alpha)\right\}\right\}, \\
& \left.\max \left\{I_{\dot{A}}^{u}(\alpha), \min \left\{I_{\dot{B}}^{u}(\alpha), I_{\dot{C}}^{u}(\alpha)\right\}\right\}\right], \\
& {\left[\max \left\{F_{\dot{A}}^{l}(\alpha), \min \left\{F_{\dot{B}}^{l}(\alpha), F_{\dot{C}}^{l}(\alpha)\right\}\right\},\right.} \\
& \left.\left.\max \left\{F_{\dot{A}}^{u}(\alpha), \min \left\{F_{\dot{B}}^{u}(\alpha), F_{\dot{C}}^{u}(\alpha)\right\}\right\}\right]\right\rangle
\end{aligned}
$$

$\mid \alpha \in X\}$

$$
\begin{aligned}
=\{\langle\alpha, & {\left[\max \left\{\min \left\{T_{\dot{A}}^{l}(\alpha), T_{\dot{B}}^{l}(\alpha)\right\}, \min \left\{T_{\dot{A}}^{l}(\alpha), T_{\dot{C}}^{l}(\alpha)\right\}\right\},\right.} \\
& \left.\max \left\{\min \left\{T_{\dot{A}}^{u}(\alpha), T_{\dot{B}}^{u}(\alpha)\right\}, \min \left\{T_{\dot{A}}^{u}(\alpha), T_{\dot{C}}^{u}(\alpha)\right\}\right\}\right], \\
& {\left[\min \left\{\max \left\{I_{\dot{A}}^{l}(\alpha), I_{\dot{B}}^{l}(\alpha)\right\}, \max \left\{I_{\dot{A}}^{l}(\alpha), I_{\dot{C}}^{l}(\alpha)\right\}\right\},\right.} \\
& \left.\min \left\{\max \left\{I_{\dot{A}}^{u}(\alpha), I_{\dot{B}}^{u}(\alpha)\right\}, \max \left\{I_{\dot{A}}^{u}(\alpha), I_{\dot{C}}^{u}(\alpha)\right\}\right\}\right], \\
& {\left[\min \left\{\max \left\{F_{\dot{A}}^{l}(\alpha), F_{\dot{B}}^{l}(\alpha)\right\}, \max \left\{F_{\dot{A}}^{l}(\alpha), F_{\dot{C}}^{l}(\alpha)\right\}\right\},\right.} \\
& \left.\left.\min \left\{\max \left\{F_{\dot{A}}^{u}(\alpha), F_{\dot{B}}^{u}(\alpha)\right\}, \max \left\{F_{\dot{A}}^{u}(\alpha), F_{\dot{C}}^{u}(\alpha)\right\}\right\}\right]\right\rangle
\end{aligned}
$$

$\mid \alpha \in X\}$

$=(\dot{A} \widehat{\cap} \dot{B}) \widehat{U}(\dot{A} \hat{\cap} \dot{C})$

iv. It is straightforward.

Theorem 3.7 Let $\dot{A}$ and $\dot{B}$ be two ivns on $X$. Then,

i. $\overline{(\dot{A} \hat{\cap} \dot{B})}=\overline{\dot{A}} \widehat{\cup} \bar{B}$

ii. $\overline{(\dot{A} \widehat{\cup} \dot{B})}=\overline{\dot{A}} \hat{\cap} \bar{B}$

Proof. Let's consider ivns $\dot{A}$ and $\dot{B}$ on $X$ as follows.

$\dot{A}=\left\{\left\langle\alpha,\left[T_{\dot{A}}^{l}(\alpha), T_{\dot{A}}^{u}(\alpha)\right)\right],\left[I_{\dot{A}}^{l}(\alpha), I_{\dot{A}}^{u}(\alpha)\right],\left[F_{\dot{A}}^{l}(\alpha), F_{\dot{A}}^{u}(\alpha)\right]\right\rangle$

$\mid \alpha \in X\}$ 
$\dot{B}=\left\{\left\langle\alpha,\left[T_{\dot{B}}^{l}(\alpha), T_{\dot{B}}^{u}(\alpha)\right],\left[I_{\dot{B}}^{l}(\alpha), I_{\dot{B}}^{u}(\alpha)\right],\left[F_{\dot{B}}^{l}(\alpha), F_{\dot{B}}^{u}(\alpha)\right]\right\rangle\right.$

$\mid \alpha \in X\}$

i. For all $x \in X$

$\dot{A} \widehat{\cap} \dot{B}=\left\{\left\langle\alpha,\left[\min \left\{T_{\dot{A}}^{l}(\alpha), T_{\dot{B}}^{l}(\alpha)\right\}, \min \left\{T_{\dot{A}}^{u}(\alpha), T_{\dot{B}}^{u}(\alpha)\right\}\right]\right.\right.$,

$$
\begin{gathered}
{\left[\max \left\{I_{\dot{A}}^{l}(\alpha), I_{\dot{B}}^{l}(\alpha)\right\}, \max \left\{I_{\dot{A}}^{u}(\alpha), I_{\dot{B}}^{u}(\alpha)\right\}\right],} \\
\left.\left[\max \left\{F_{\dot{A}}^{l}(\alpha), F_{\dot{B}}^{l}(\alpha)\right\}, \max \left\{F_{\dot{A}}^{u}(\alpha), F_{\dot{B}}^{u}(\alpha)\right\}\right]\right\rangle
\end{gathered}
$$

$\mid \alpha \in X\}$

$\overline{(\dot{A} \hat{\cap} \dot{B})}=\left\{\left\langle\alpha,\left[\max \left\{F_{\dot{A}}^{l}(\alpha), F_{\hat{B}}^{l}(\alpha)\right\}, \max \left\{F_{\dot{A}}^{u}(\alpha), F_{\dot{B}}^{u}(\alpha)\right\}\right]\right.\right.$,

\section{Conclusions}

Many types of operations are available in interval-valued neutrosophic sets, which not only handle uncertainty but also preserve the data in a very nice way. In the present paper we have introduced some operations. These operations are very flexible and useful generalizations of existing operations available in interval-valued neutrosophic set theory. It is hoped these newly introduced concepts will find their applications in many fields soon.

\section{References}

[1] Zadeh, L. A. (1965). Fuzzy Sets, Information and Control, 8, 338-353.

[2] Atanassov, K. T. (1986). Intuitionistic fuzzy sets, Fuzzy Sets and Systems, 20, 87-96.

[3] Smarandache, F. (2006). Neutrosophic set-a generalization of the intuitionistic fuzzy set, In Proceedings of 2006 IEEE International Conference on Granular Computing, Georgia State University, Atlanta, 38-42.

[4] Wang, H., Smarandache, F., Zhang, Y. Q. and Sunderraman, R. (2010). Single valued neutrosophic sets, Multispace and Multistructure, 4, 410-413.

[5] Zhang, H. Y., Wang, J. Q. and Chen, X. H. (2014). Interval neutrosophic sets and their application in multicriteria decision making problems, The Scientific World Journal, 3, 649-653.

[6] Lupianez, F. G. (2009). Interval Neutrosophic Sets and Topology, Kybernetes, 38, 621-624.

[7] Zadeh, L. A. (1975). The concept of linguistic variable and its application to approximate reasoning-I, Information Science, 8(3), 199-249.

[8] Smarandache, F. (2005). Neutrosophic set- a generalization of the intuitionistic fuzzy set, International Journal of Pure and Applied Mathematics, 24(3), 287-297.

[9] Wang, H., Smarandache, F., Zhang, Y. Q. and Sunderraman, R. (2005). Interval Neutrosophic Sets and Logic: Theory and Applications in Computing, In: Neutrosophic book series, Hexis, Arizona.

$$
\begin{aligned}
& {\left[\min \left\{1-I_{\dot{A}}^{u}(\alpha), 1-I_{\dot{B}}^{u}(\alpha)\right\}, \min \left\{1-I_{\dot{A}}^{u}(\alpha), 1-I_{\dot{B}}^{l}(\alpha)\right\}\right],} \\
& \left.\left.\left.\min \left\{T_{\dot{A}}^{l}(\alpha), T_{\dot{B}}^{l}(\alpha)\right\}, \min \left\{T_{\dot{A}}^{u}(\alpha), T_{\dot{B}}^{u}(\alpha)\right\}\right]\right\rangle \mid \alpha \in X\right\} \\
& =\left\{\left\langle\alpha,\left[F_{\dot{A}}^{l}(\alpha), F_{\dot{A}}^{u}(\alpha)\right],\left[1-I_{\dot{A}}^{u}(\alpha), 1-I_{\dot{A}}^{l}(\alpha)\right],\left[T_{\dot{A}}^{l}(\alpha), T_{\dot{A}}^{u}(\alpha)\right]\right\rangle\right. \\
& \mid \alpha \in X\} \widehat{\cup}\left\{\left\langle\alpha,\left[F_{\dot{B}}^{l}(\alpha), F_{\dot{B}}^{u}(\alpha)\right],\left[1-I_{\dot{B}}^{u}(\alpha), 1-I_{\dot{B}}^{l}(\alpha)\right],\right.\right. \\
& \left.\left.\left[T_{\dot{B}}^{l}(\alpha), T_{\dot{B}}^{u}(\alpha)\right]\right\rangle \mid \alpha \in X\right\} \\
& =\overline{\dot{A}} \cup \overline{\dot{B}} \\
& \text { ii. It is straightforward. }
\end{aligned}
$$

